\title{
Cholesterol toxicity in pancreatic islets from LDL receptor-deficient mice. Reply to: de Souza JC, de Oliveira CAM, Carneiro EM et al. [letter]
}

\author{
J. K. Kruit • L. R. Brunham • C. B. Verchere • \\ M. R. Hayden
}

Received: 14 July 2010 /Accepted: 15 July 2010 / Published online: 26 August 2010

(C) Springer-Verlag 2010

Keywords ABCA1 - Beta cell function .

Hypercholesterolaemia $\cdot$ Islets $\cdot$ LDL receptor

\author{
Abbreviations \\ FH Familial hypercholesterolaemia \\ LDLr LDL receptor
}

To the Editor: In their letter [1], de Souza and colleagues point out important differences between our study [2] and their subsequent report on the role of the LDL receptor (LDLr) in islet cholesterol handling. In contrast to their data [3], we did not detect a significant difference between islet cholesterol levels in mice lacking $\operatorname{LDLr}\left(\mathrm{Ldlr}^{--}\right)$and control mice [2]. Accordingly, we found no difference in glucose tolerance or insulin secretion in $A b c a 1^{\mathrm{fl} / \mathrm{fl}} ; \mathrm{Ldlr}^{+/+}$mice compared with

J. K. Kruit • L. R. Brunham • M. R. Hayden $(\bowtie)$

Centre for Molecular Medicine and Therapeutics,

Child and Family Research Institute,

Department of Medical Genetics, University of British Columbia,

950 West 28th Ave,

Vancouver, BC, Canada V5Z 4H4

e-mail: mrh@cmmt.ubc.ca

C. B. Verchere

Child and Family Research Institute,

Department of Surgery, University of British Columbia,

Vancouver, BC, Canada

\section{B. Verchere}

Child and Family Research Institute,

Department of Pathology and Laboratory Medicine,

University of British Columbia,

Vancouver, BC, Canada
Abcal ${ }^{\mathrm{f} / / \mathrm{fl}} ; \mathrm{Ldlr}^{-/-}$mice on a chow diet. This is in agreement with previous literature showing no effect of LDLr deficiency on fasting glucose levels and glucose tolerance in chow-fed mice and slightly improved glucose tolerance in mice fed a Western-type diet [4, 5]. At this point the reasons for the discordant results obtained by Bonfleur and colleagues are not clear, as both groups used mice bred on a pure C57B16 background at similar ages (16 weeks). However, the results of both groups point to the ability of circulating and cellular cholesterol to significantly influence islet function.

If islet cholesterol levels in $L d l r^{-/}$mice are the result of a gradient-driven cholesterol flow from the plasma to the cell membranes associated with de novo cholesterol synthesis, as suggested by de Souza et al. [1], increased plasma cholesterol levels would result in increased cholesterol levels in islets. However, a Western-type diet did not result in increased islet cholesterol levels in islets isolated from $\mathrm{Ldlr}^{-1-}$ mice, despite the presence of severe hypercholesterolaemia. In addition, we show that LDL uptake is decreased in islets lacking LDLr [2]. Therefore, we propose that lack of the LDLr protects beta cells from accumulation of cholesterol in a hypercholesterolaemic environment.

In agreement with our findings in $L d l r^{-/}$mice, patients with familial hypercholesterolaemia $(\mathrm{FH})$, which is either caused by mutations in $L D L R$ or the gene encoding apolipoprotein B100, appear to be protected from type 2 diabetes. In a case-control study, the prevalence of type 2 diabetes in $\mathrm{FH}$ patients was less than half that in non-FH controls, despite the presence of markedly increased plasma cholesterol levels in these patients $[6,7]$.

In addition, a recent meta-analysis of several randomised statin trials indicated that statin therapy is associated with a slightly increased risk of development of diabetes [8]. The 
reasons for this are unknown, but it is provocative to consider that statins, which suppress de novo cellular cholesterol synthesis and thereby increase LDLr expression in beta cells [2], may actually lead to elevated levels of islet cholesterol. This hypothesis needs testing, but if proven, would provide a potential mechanism for the increased risk of diabetes in patients treated with these medications.

Duality of interest The authors declare that there is no duality of interest associated with this manuscript.

\section{References}

1. de Souza JC, de Oliveira CAM, Carneiro EM, Boschero AC, de Oliveira HCF (2010) Cholesterol toxicity in pancreatic islets from LDL receptordeficient mice. Diabetologia. doi:10.1007/s00125-010-1868-8

2. Kruit JK, Kremer PHC, Dai L, et al. (2010) Cholesterol efflux via ATP-binding cassette transporter A1 (ABCA1) and cholesterol uptake via the LDL receptor influences cholesterol-induced impairment of beta cell function in mice. Diabetologia 53: 1110 1119

3. Bonfleur ML, Vanzela EC, Ribeiro RA et al (2010) Primary hypercholesterolaemia impairs glucose homeostasis and insulin secretion in low-density lipoprotein receptor knockout mice independently of high-fat diet and obesity. Biochim Biophys Acta 1801:183-190

4. Coenen KR, Gruen ML, Hasty AH (2007) Obesity causes very low density lipoprotein clearance defects in low-density lipoprotein receptor-deficient mice. J Nutr Biochem 18:727-735

5. Karagiannides I, Abdou R, Tzortzopoulou A, Voshol PJ, Kypreos KE (2008) Apolipoprotein E predisposes to obesity and related metabolic dysfunctions in mice. FEBS J 275:47964809

6. Skoumas I, Masoura C, Pitsavos C et al (2007) Evidence that nonlipid cardiovascular risk factors are associated with high prevalence of coronary artery disease in patients with heterozygous familial hypercholesterolemia or familial combined hyperlipidemia. Int $\mathrm{J}$ Cardiol 121:178-183

7. Vohl MC, Gaudet D, Moorjani S et al (1997) Comparison of the effect of two low-density lipoprotein receptor class mutations on coronary heart disease among French-Canadian patients heterozygous for familial hypercholesterolaemia. Eur J Clin Invest 27:366-373

8. Sattar N, Preiss D, Murray HM et al (2010) Statins and risk of incident diabetes: a collaborative meta-analysis of randomized statin trials. Lancet 375:735-742 